\title{
Correction: Men with spinal cord injury have a smaller prostate volume than age-matched able-bodied men: a meta-analysis of case-control studies
}

Parisi Antonio, Totaro Maria, Castellini Chiara, D’ Andrea Settimio (D), Tienforti Daniele, Palazzi Sara, D’ Amato Federica, Muselli Mario, Francavilla Sandro and Barbonetti Arcangelo

(c) The Author(s), under exclusive licence to International Spinal Cord Society 2021

Spinal Cord (2021) 59:1219; https://doi.org/10.1038/s41393-021-00718-1

Correction to: Spinal Cord https://doi.org/10.1038/s41393-02100712-7
The original version of this article unfortunately contained a mistake. The names and surnames of the authors were inverted. The authors apologize for the error. The original article has been corrected. 